Tuberc Respir Dis 2012;73:107-114

Copyright(C)2012. The Korean Academy of Tuberculosis and Respiratory Diseases. All rights reserved.

\title{
Analysis of Patients with Hemoptysis in a Tertiary Referral Hospital
}

Bo Ram Lee, M.D., Jin Yeong Yu, M.D., Hee Jung Ban, M.D., In Jae Oh, M.D., Kyu Sik Kim, M.D., Yong Soo Kwon, M.D., Yu II Kim, M.D., Young Chul Kim, M.D., Sung Chul Lim, M.D.

Department of Internal Medicine, Chonnam National University Hospital, Chonnam National University Medical School, Gwangju, Korea

Background: This study attempted to investigate the main causes of hemoptysis, the type of examinations used for diagnosis, the treatment modalities and outcomes.

Methods: A retrospective study was conducted on the medical records of 221 patients admitted to the Chonnam National University Hospital, between January 2005 and February 2010, with hemoptysis.

Results: Bronchiectasis (32.6\%), active pulmonary tuberculosis (18.5\%), fungus ball (10.8\%), and lung cancer (5.9\%) accounted for most causes of hemoptysis. Computed tomography scan was the most sensitive diagnostic test when employed alone, with positive yield of $93.2 \%$. There were 161 cases of conservative treatment $(72.9 \%), 42$ cases of bronchial artery embolization (BAE) (19.0\%), and 18 cases of surgery (8.1\%). Regarding the amount of hemoptysis, 70 cases, out of 221 cases, were mild (31.5\%), 36 cases moderate (16.2\%), and 115 cases massive hemoptysis (52.0\%). Most of the patients were treated conservatively, but if there was more bleeding present, BAE or surgery was more commonly performed than the conservative treatment $(\mathrm{p} \leq 0.0001)$. In the multivariate model, severe hemoptysis and lung cancer were independently associated with short-term recurrence. BAE was independently associated with long-term recurrence, and lung cancer was associated with in-hospital mortality. The overall in-hospital mortality rate was $11.3 \%$.

Conclusion: Hemoptysis is a common symptom with a good prognosis in most cases. However, patients exhibiting massive bleeding or those with malignancy had a poorer prognosis. In-hospital mortality was strongly related to the cause, especially in lung cancer.

Key Words: Hemoptysis; Etiology; Diagnosis; Therapeutics; Treatment Outcome

\section{Introduction}

Hemoptysis is the expectoration of blood from the airways. Hemoptysis results from destroyed lung parenchyma, for which the most common causes are tuberculosis and bronchiectasis in Korea ${ }^{1,2}$, and bronchitis and bronchial cancer in other countries ${ }^{3,4}$.

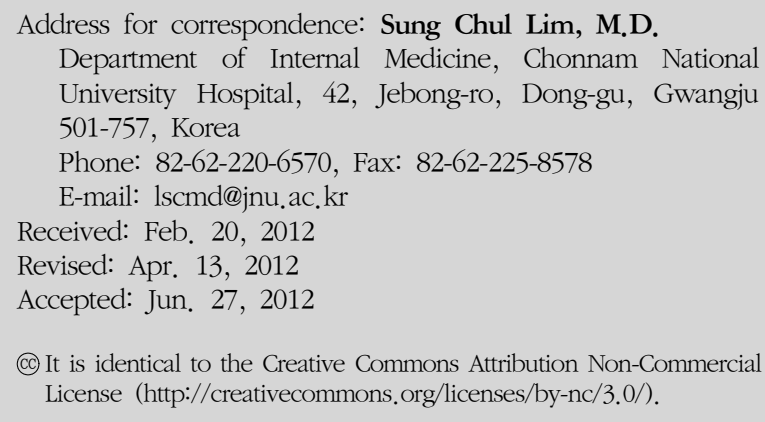

Hemoptysis severity ranges from asymptomatic condition to airway obstruction due to hemoptysis. While only the severe (range, $\geq 100 \mathrm{~mL} / 24 \mathrm{hr} \sim \geq 1,000$ $\mathrm{mL} / 24 \mathrm{hr}^{2,4-8}$ ) expectoration of pure blood is called hemoptysis, it generally includes blood-tinged or bloodstreaked sputum ${ }^{1}$. Typically, $\geq 600 \mathrm{~mL} / 24 \mathrm{hr}$ is considered as massive hemoptysis ${ }^{9,10}$, but $\geq 100 \mathrm{~mL} / 24 \mathrm{hr}$ is also sometimes referred to as massive hemoptysis ${ }^{6,7}$.

Conservative treatment, bronchial artery embolization (BAE), and surgical treatment are available. However, it is often the case that conservative treatment fails to control bleeding; on the other hand, surgical methods, such as pneumonectomy, are difficult to perform and are associated with high postoperative in-hospital mortality. With good immediate success rate for hemostasis, BAE is effective, but its long-term success rate is 
BR Lee et al: Analysis of patients with hemoptysis in a tertiary referral hospital

undesirable $e^{3,11,12}$. A large number of studies have been conducted on the prognostic factors influencing the curative effects of $\mathrm{BAE}$, but so far with no conclusive results $^{5,13}$. It is also difficult to determine the appropriate treatment method because most of hemoptysis patients have chronic lung disease and poor performance status.

In this study, we investigated the clinical characteristics and etiology of hemoptysis in a hospital-based series of Korean patients. We describe the type of examinations used for diagnosis, the treatment modalities, and outcomes.

\section{Materials and Methods}

We reviewed the medical records of patients admitted to the Chonnam National University Hospital between January 2005 and February 2010 with hemoptysis.

The inclusion criteria were as follows: diagnosis of hemoptysis; patients treated with hemostatics (Hemocoagulase, Botropase; Hanlim Pharm. Co., Yongin, Korea); patients who underwent chest computed tomography (CT); and patients who were actually treated for hemoptysis. Based on the examination of the medical and radiotherapy records, an analysis was made on the etiology of disease, smoking history, past medical history, physical examination, chest radiography and CT, sputum smear test and culture, bronchoscopy and histopathological examination, bronchial arteriography, and postoperative histopathological examination.

In this study, hemoptysis was divided into mild $(<30$ $\mathrm{mL} / 24 \mathrm{hr}$ ), moderate ( $\geq 30$ and $<100 \mathrm{~mL} / 24 \mathrm{hr}$ ), and severe or massive ( $\geq 100 \mathrm{~mL} / 24 \mathrm{hr}$ ) according to Fidan et al. ${ }^{6}$.

Active pulmonary tuberculosis was defined as positivity for acid-fast bacillus (AFB) on bronchoscopy aspirate smear test, positivity for Mycobacterium tuberculosis on tuberculosis culture, or positivity on tuberculosis polymerase chain reaction (TB-PCR) test. Bronchiectasis was diagnosed based on chest CT imaging, and lung cancer was diagnosed based on histopathological examination.

When chest CT, bronchoscopy, or bronchial arteriog- raphy could detect the bleeding site or cause, it was defined as being helpful for diagnosis.

The follow-up period of patients was based on the last day of visit, and the success rate of hemoptysis control was determined by dividing the term largely into short-term and long-term.

Short-term results were assessed based on careful observation of patients for 1 month (30 days or less) after first-line treatment and were classified into two categories: success, indicating complete cessation of hemoptysis during 1 month (30 days or less); and recurrence ${ }^{12}$. Treatment failure was defined when moderate bleeding or worse continued three days after first-line treatment, and included the cases where the second-line treatment was performed, or the patient was transferred, discharged, or died without second-line treatment. When a patient was discharged because of mild hemoptysis three days after first-line treatment and did not need second-line treatment for hemoptysis, it was not considered as treatment failure. Recurrence included treatment failure, and was defined as single or multiple episodes of hemoptysis. Long-term results were evaluated in patients who could be followed for at least 1 month. Successful long-term control was defined as the successful control of hemoptysis without recurrence for the follow-up period (longer than 1 month). The same patient could be included in both short-term and long-term groups. In-hospital mortality was assessed in patients with long-term follow-up.

In addition, the patients were divided into conservative treatment, BAE, and surgical treatment group, depending on their first-line treatment regardless of the cause. Follow-up period of conservative treatment was based on the day of hospital admission. To compare the differences between short-term control, long-term control, in-hospital mortality, and duration of hospitalization, we performed statistical analyses.

For the statistical analysis, the SPSS version 18.0 (SPSS Inc., Chicago, IL, USA) program was used. The continuous variable data were presented with mean \pm standard deviation. In addition, ANOVA test was performed to compare the hospitalization duration of each 
group and the chi-square test was carried out for the analysis of outcomes. A p-value less than 0.05 was considered to be statistically signifiant. To verify the compounding factors between the risk factors whose characters were diverse confounding variables, we did multivariate analysis using logistic regression analysis with 95\% confidence interval.

\section{Results}

\section{Clinical characteristics}

Of a total of 221 patients, $145(65.6 \%)$ were male and $76(34.4 \%)$ female with average age of $57.9 \pm 15.6$.

As a past medical history, 58 patients had high blood pressure (26.2\%), 28 patients diabetes (12.7\%), 13 patients both high blood pressure and diabetes (5.9\%), and 80 patients had the history of pulmonary tuberculosis (36.2\%).

The follow-up period was $14.12 \pm 12.59$ months.

\section{The causes and treatment methods of hemoptysis}

Regarding the amount of hemoptysis, 70 cases out of 221 cases were mild (31.5\%), 36 cases moderate (16.2\%), and 115 cases massive hemoptysis (52.0\%).

As for the causes, 72 cases were bronchiectasis (32.6\%), 41 cases active pulmonary tuberculosis (18.5\%), 24 cases fungus ball (10.8\%), 13 cases lung cancer (5.9\%), 45 cases other causes (20.3\%), and 26 cases unknown cause (11.7\%). Other causes included lung abscess, necrotizing pneumonia, pulmonary thromboembolism, pulmonary sequestration, arteriovenous malformation, catamenial pneumothorax, etc.

As for the causes of 115 massive hemoptysis patients, 39 cases were bronchiectasis (39.9\%), the highest frequency, followed by 24 cases of active pulmonary tuberculosis (20.9\%), 16 cases of pulmonary fungus ball (13.9\%), 7 cases of lung cancer (6.1\%), 18 cases of other causes (15.7\%), and 11 cases of unknown causes (9.6\%). The causes of mild and moderate hemoptysis are as follows, with no significant difference $(\mathrm{p}=0.47)$. Most of the patients treated conservatively, but if there was more bleeding present, BAE or surgery was more commonly performed than conservative treatment $(\mathrm{p} \leq$ 0.0001) (Table 1).

\section{Diagnostic evaluation}

Chest CT was performed for all patients (221 cases), of which pulmonary lesions were detected in 206 cases (93.2\%). Bronchoscopy was performed for 106 cases (48.0\%), of which 64 cases $(60.0 \%)$ were helpful for diagnosis. Bronchial arteriography was performed for 44 cases (18.6\%), of which 41 cases (93\%) were helpful for diagnosis.

When an active pulmonary tuberculosis was suspicious from chest CT, a test for sputum or bronchoscopy aspirate was conducted. Active pulmonary tuberculosis was defined as patients who were positive for AFB from microscopic examination of stained smears or positive

Table 1. The causes and treatment methods of hemoptysis

\begin{tabular}{llccc}
\hline & & Mild & Moderate & Severe \\
\hline Cause & Bronchiectasis & $22(31.4)$ & $11(30.6)$ & $39(33.9)$ \\
& Active pul TB & $11(15.7)$ & $6(16.7)$ & $24(20.9)$ \\
& Lung cancer & $5(7.1)$ & $1(2.8)$ & $7(6.1)$ \\
& Fungus ball & $3(4.3)$ & $5(13.9)$ & $16(13.9)$ \\
& Idiopathic & $10(14.3)$ & $8(22.9)$ & $11(9.6)$ \\
Treatment & Miscellaneous & $19(27.1)$ & $30(83.3)$ & $18(15.7)$ \\
& Conservative tx & $62(88.6)$ & $5(13.9)$ & $32(60.0)$ \\
& BAE & $5(7.1)$ & $1(2.8)$ & $14(12.2)$ \\
\hline
\end{tabular}

Values are presented as number (\%).

pul TB: pulmonary tuberculosis; tx: treatment; BAE: bronchial artery embolization. 
BR Lee et al: Analysis of patients with hemoptysis in a tertiary referral hospital

Table 2. The methodology of diagnosis

\begin{tabular}{|c|c|c|c|c|c|c|}
\hline & \multicolumn{2}{|c|}{ Chest CT } & \multicolumn{2}{|c|}{ Bronchoscopy } & \multicolumn{2}{|c|}{ Bronchial arteriography } \\
\hline & Performed & Positive result & Performed & Positive result & Performed & Positive result \\
\hline Bronchiectasis & $72(100)$ & $72(100)$ & $34(47)$ & $24(70)$ & $22(30)$ & $21(95)$ \\
\hline Fungus ball & $24(100)$ & $24(100)$ & $18(75)$ & 4 (22) & 4 (16) & 4 (100) \\
\hline Lung cancer & $13(100)$ & $13(100)$ & $6(48)$ & $6(100)$ & $0(0)$ & $0(0)$ \\
\hline Active pul TB & $41(100)$ & $41(100)$ & $14(34)$ & $10(71)$ & $12(29)$ & $12(100)$ \\
\hline Miscellaneous & $45(100)$ & $44(98)$ & $19(42)$ & $14(73)$ & 4 (8) & $4(100)$ \\
\hline Idiopathic & $26(100)$ & $12(46)$ & $15(58)$ & $6(40)$ & $2(7)$ & $0(0)$ \\
\hline Total & $221(100)$ & 206 (93.2) & $106(48)$ & $64(60)$ & $44(18.6)$ & 41 (93) \\
\hline
\end{tabular}

Values are presented as number (\%).

CT: computed tomography; pul TB: pulmonary tuberculosis.

for mycobacterium tuberculosis from tuberculosis culture, or positive for TB-PCR. Among 41 cases of active pulmonary tuberculosis, all three kinds of test-positive cases were 5, two kinds of test-positive cases were 10 ( 1 case positive for culture and AFB, 1 case positive for culture and PCR, 8 cases positive for PCR and AFB), and 26 cases were positive for only one kind of test ( 1 case positive for only culture, 5 cases positive for only PCR, 20 cases positive for only AFB).

All 72 cases of bronchiectasis were diagnosed by chest CT. Bronchoscopy was performed for 34 cases (47.2\%), of which 24 cases (70\%) showed abnormalities. Bronchial arteriography was performed for 22 cases (30\%), of which 21 cases (95\%) were detected for the bleeding site.

All 24 cases of fungus ball were also diagnosed by chest CT. Bronchoscopy was performed for 18 cases (75\%), of which 4 cases (22\%) were detected for active bleeding from the bronchus. Bronchial arteriography was performed for 4 cases (16\%), of which artery embolization was performed for all 4 cases after checking offending vessels.

In lung cancer, all 13 cases showed abnormalities from chest CT. Bronchoscopy was performed for 6 cases $(46 \%)$ and all the 6 cases were helpful for the diagnosis. The final diagnosis was made by sputum cytology test, biopsy, or percutaneous fine-needling.

Of the 26 cases with unknown cause, 12 cases showed non-local abnormalities (e.g., hemorrhagic as- pirate, etc.) from chest CT and 14 cases were normal. Bronchoscopy was performed for 15 cases, of which 6 cases showed the evidence of previous bleeding without any clear cause (Table 2).

\section{Treatment outcome according to the amount of hemoptysis}

In the case of mild hemoptysis, 67 cases (95.7\%) out of 70 cases were controlled during 1 month, while 34 cases (94.4\%) out of 36 cases were controlled in moderate hemoptysis. In the case of massive hemoptysis, 94 cases $(81.7 \%)$ out of 115 cases were controlled, showing a higher failure or recurrence rate compared to mild and moderate hemoptysis $(\mathrm{p}=0.008)$ (Table 3).

There were 161 cases of conservative treatment (72.9\%), 42 cases of BAE (19.0\%), 18 cases of surgical treatment $(8.1 \%)$.

Of the 221 cases, only 168 cases were available for long-term follow-up because the 53 cases of follow-up loss were excluded from the analysis.

\section{Treatment outcome according to the treatment method}

The short-term and long-term control, in-hospital mortality rates were investigated according to treatment method. In the short-term control, the success rate of conservative treatment group was $88.2 \%$, BAE group $92.9 \%$, surgical treatment group 88.9\%, showing that the success rate of BAE was significantly high $(p=0.04)$. As 
Tuberculosis and Respiratory Diseases Vol. 73. No. 2, Aug. 2012

for the success rate of the long-term control, the conservative treatment group was 80.2\%, BAE group 63.6\%, surgical treatment group $78.6 \%$, without any significant difference $(p=0.135)$. In addition, there was no statistical difference in the in-hospital mortality $(\mathrm{p}=0.873)$ (Table 3).

Table 3. Treatment outcome according to the amount of hemoptysis, treatment method, and leading cause

\begin{tabular}{|c|c|c|c|c|c|c|c|c|c|}
\hline & \multicolumn{2}{|c|}{ Short-term control } & \multirow{2}{*}{ p-value } & \multicolumn{2}{|c|}{ Long-term control } & \multirow{2}{*}{ p-value } & \multicolumn{2}{|c|}{ In-hospital mortality } & \multirow{2}{*}{$p$-value } \\
\hline & Success & Recurrence & & Success & Recurrence & & Alive & Death & \\
\hline Amount & & & 0.008 & & & 0.625 & & & 0.117 \\
\hline Mild & $67(95.7)$ & $3(4.3)$ & & $43(76.8)$ & $13(23.2)$ & & 52 (92.9) & $4(7.1)$ & \\
\hline Moderate & $34(94.4)$ & $2(5.6)$ & & $21(84.0)$ & $4(16.0)$ & & $24(96.0)$ & $1(4.0)$ & \\
\hline Severe & $94(81.7)$ & $21(18.3)$ & & $65(74.7)$ & $22(25.3)$ & & $73(83.9)$ & $14(16.1)$ & \\
\hline Treatment & & & 0.04 & & & 0.135 & & & 0.873 \\
\hline Conservative $t x$ & $142(88.2)$ & $19(11.8)$ & & $97(80.2)$ & $24(19.8)$ & & $107(88.4)$ & $14(11.6)$ & \\
\hline BAE & 39 (92.9) & $3(7.1)$ & & $21(63.6)$ & $12(36.4)$ & & $29(87.9)$ & $4(12.1)$ & \\
\hline Surgery & $16(88.9)$ & $2(11.1)$ & & $11(78.6)$ & $3(21.4)$ & & $13(92.9)$ & $1(7.1)$ & \\
\hline Cause & & & 0.055 & & & 0.281 & & & 0.001 \\
\hline Bronchiectasis & $66(91.7)$ & $6(8.3)$ & & $45(77.6)$ & $13(22.4)$ & & $56(96.6)$ & $2(3.4)$ & \\
\hline Active pul TB & $36(87.8)$ & 5 (12.2) & & $28(84.8)$ & $5(15.2)$ & & $30(90.9)$ & $3(9.1)$ & \\
\hline Fungus ball & 19 (79.2) & $5(20.8)$ & & $10(76.9)$ & $3(23.1)$ & & $10(76.9)$ & $3(23.1)$ & \\
\hline Lung cancer & 9 (69.2) & $4(30.8)$ & & $8(80.0)$ & $2(20.0)$ & & $5(50.0)$ & $5(50.0)$ & \\
\hline Idiopathic & $26(100)$ & $0(0)$ & & $14(87.5)$ & $2(12.5)$ & & $15(93.8)$ & $1(6.3)$ & \\
\hline Miscellaneous & 39 (86.7) & $6(13.3)$ & & 24 (63.2) & $14(36.8)$ & & 33 (86.8) & 5 (13.2) & \\
\hline Total & 195 (88.2) & $26(11.8)$ & & $129(76.8)$ & $39(23.2)$ & & $149(88.7)$ & $19(11.3)$ & \\
\hline
\end{tabular}

Values are presented as number (\%).

tx: treatment; BAE: bronchial artery embolization; pul TB: pulmonary tuberculosis.

Table 4. Multivariate analysis of different variables

\begin{tabular}{|c|c|c|c|c|c|c|c|}
\hline & \multirow{2}{*}{ Variables } & \multicolumn{2}{|c|}{ Short-term control } & \multicolumn{2}{|c|}{ Long-term control } & \multicolumn{2}{|c|}{ In-hospital mortality } \\
\hline & & OR (95\% Cl) & $p$-value & OR (95\% Cl) & $p$-value & OR (95\% Cl) & p-value \\
\hline \multirow[t]{2}{*}{ Gender } & Female & 1 & & 1 & & 1 & \\
\hline & Male & $0.84(0.39 \sim 3.15)$ & 0.835 & $2.00(0.91 \sim 4.39)$ & 0.083 & $0.77(0.24 \sim 2.43)$ & 0.656 \\
\hline \multirow[t]{2}{*}{ Age } & $<50 \mathrm{yr}$ & 1 & & 1 & & 1 & \\
\hline & $\geq 50 \mathrm{yr}$ & $10.99(1.36 \sim 88.97)$ & 0.025 & $1.413(0.58 \sim 3.47)$ & 0.451 & $2.23(0.43 \sim 11.60)$ & 0.339 \\
\hline \multirow[t]{3}{*}{ Amount } & Mild & 1 & & 1 & & 1 & \\
\hline & Moderate & $1.31(0.20 \sim 8.49)$ & 0.777 & $0.61(0.16 \sim 2.26)$ & 0.459 & $0.61(0.06 \sim 6.64)$ & 0.687 \\
\hline & Severe & $5.93(1.61 \sim 21.79)$ & 0.007 & $1.14(0.46 \sim 2.85)$ & 0.779 & $3.64(0.90 \sim 14.77)$ & 0.071 \\
\hline \multirow[t]{3}{*}{ Treatment } & Conservative tx & 1 & & 1 & & 1 & \\
\hline & $\mathrm{BAE}$ & $0.93(0.32 \sim 2.76)$ & 0.899 & $2.87(1.09 \sim 7.55)$ & 0.032 & $1.29(0.33 \sim 5.03)$ & 0.717 \\
\hline & Surgery & $0.23(0.03 \sim 1.94)$ & 0.177 & $0.78(0.19 \sim 3.23)$ & 0.734 & $0.37(0.04 \sim 3.30)$ & 0.370 \\
\hline \multirow[t]{6}{*}{ Cause } & Idiopathic & 1 & & 1 & & 1 & \\
\hline & Active pul TB & $3.02(0.32 \sim 28.61)$ & 0.336 & $0.79(0.07 \sim 9.37)$ & 0.854 & $1.33(0.12 \sim 14.50)$ & 0.817 \\
\hline & Lung cancer & $11.34(1.03 \sim 124.46)$ & 0.047 & $1.73(0.10 \sim 28.59)$ & 0.703 & $24.49(1.98 \sim 303.07)$ & 0.013 \\
\hline & Bronchiectasis & $2.22(0.24 \sim 20.20)$ & 0.478 & $1.47(0.14 \sim 15.7)$ & 0.750 & $0.56(0.05 \sim 6.84)$ & 0.650 \\
\hline & Fungus ball & $6.26(0.64 \sim 61.19)$ & 0.115 & $1.66(0.12 \sim 23.65)$ & 0.707 & $4.13(0.35 \sim 48.49)$ & 0.260 \\
\hline & Miscellaneous & $4.88(0.53 \sim 44.80)$ & 0.161 & $3.74(0.33 \sim 41.86)$ & 0.285 & $3.14(0.32 \sim 30.83)$ & 0.327 \\
\hline
\end{tabular}

OR: odds ratio; $\mathrm{Cl}$ : confidence interval; tx: treatment; BAE: bronchial artery embolization; pul TB: pulmonary tuberculosis. 


\section{Treatment outcome according to the leading cause}

In the short-term control, the success rate of bronchiectasis was $91.7 \%$, the highest, while in the longterm control, the success rate of unknown cause was the highest at $87.5 \%$, without any significant difference. In the in-hospital mortality analysis, in-hospital mortality rate of lung cancer was significantly high $(\mathrm{p}=0.001)$ (Table 3).

\section{Multivariate analysis of different variables}

In the multivariate model, amount of hemoptysis and lung cancer were independently associated with shortterm recurrence. BAE was associated with long-term recurrence, and lung cancer was associated with in-hospital mortality (Table 4).

\section{Duration of hospitalization according to treatment method}

A comparison was made for the duration of hospitalization for each treatment group. The hospitalization duration of conservative treatment was 7.4 7.0 days (range, 1 53 days), BAE group 14.9 \pm 15.8 days (range, $1 \sim 71$ days), surgical treatment group 20.9 \pm 16.3 days (range, $7 \sim 81$ days), indicating that the hospitalization duration of conservative treatment was significantly shorter than other treatment groups $(\mathrm{p}=0.005)$.

\section{Discussion}

Hemoptysis is a bleeding from the bronchial or pulmonary artery, which supply blood to the lungs, due to injury or damage induced by diseases of the bronchi or lungs ${ }^{1,2,4}$.

In most cases, hemoptysis is resolved within 24 hours in the absence of treatment; mild to moderate hemoptysis is not considered an emergency condition, and the main concern is to identify the underlying cause. Massive hemoptysis, however, can cause acute respiratory failure from the blood-filled peripheral airway and alveoli, leading to 10 60\% of in-hospital mortality from bleeding or suffocation ${ }^{7}$. For the treatment of massive hemoptysis, surgical dissection of the bleeding site would be the most effective method. However, if surgery is not possible due to ongoing hemoptysis, chronic or diffuse lung disease, pulmonary dysfunction, unresectable cancer, or rebleeding after surgery, conservative treatment is the only option ${ }^{5,14,15}$.

In this study, 161 (72.9\%) out of 221 cases had conservative treatment; bleeding was controlled in 142 (88.2\%) of those cases. During the follow-up period, 19 cases had rebleeding, of which 13 cases succeeded in controlling the bleeding with conservative treatment, 3 cases had BAE, 1 case had surgery, and 2 cases died. If bleeding was excessive, BAE or surgery were more commonly performed than conservative treatment. For the treatment of massive hemoptysis, surgery was performed in 14 cases (14\%) and BAE was performed in 32 cases $(27.8 \%)$. The remaining 69 cases (60\%) were treated conservatively. Of these, 35 cases were inoperable (poor lung function in 13 cases, hemodynamic instability in 11 cases, diffuse lesions in 7 cases, and unresectable cancer in 4 cases).

Abal et al. ${ }^{16}$ reported that the most frequent underlying disease of hemoptysis was bronchiectasis, followed by inactive pulmonary tuberculosis and active pulmonary tuberculosis. Swanson et al. ${ }^{12}$ reported bronchiectasis as the most frequent, followed by pulmonary arterial hypertension and malignant tumor. In Korea, the most common causes of hemoptysis are pulmonary tuberculosis and bronchiectasis ${ }^{1,2}$, which seems to be due to the fact that the prevalence of pulmonary tuberculosis in Korea is higher than in more developed countries. The most common cause of hemoptysis in this study was also bronchiectasis, followed by pulmonary tuberculosis, when excluding other diseases. The main cause of massive hemoptysis was bronchiectasis, too. The finding of bronchiectasis as the main cause for hemoptysis in our study is probably secondary to remote nonactive infection by $M$. tuberculosis or other infectious agents. In addition, since our study population consisted mainly of outpatients, the frequency of bronchitis patients was low.

CT has been shown to be accurate in the diagnosis 
of a wide range of bronchial abnormalities, including central tumors and peripheral lesions such as bronchiectasis $^{17,18}$. Bronchoscopy is a considerably valuable method in locating the site of bleeding, removal of obstructive clots, direct visualization of endobronchial tumors, foreign bodies, granulomas, and infiltrations. It also allows collection of histologic samples, but is not useful in detecting peripheral tumors ${ }^{19}$. Bronchial arteriography can localize potential bleeding sites and treat the bleeding by embolization ${ }^{2}$. In our study, CT had the best yield in the search for the cause of hemoptysis, and was found more valuable than bronchoscopy in the diagnosis of bronchiectasis, fungus ball, and active pulmonary tuberculosis.

Since BAE was first reported by Rémy et al. ${ }^{14}$ in 1977, it has been widely used because it can stabilize the condition of patients, gaining time for the preparation of surgery for acute hemoptysis, along with temporary treatment. The early success rate of BAE is $73 \sim$ $98 \%, 13,14,20,21$. In Korea, the early success rate is $95 \%$, and long-term success rate is $36 \%^{15}$. In our analysis, the first-line success rate of BAE was 92.9\%, while the long-term success rate was $63.6 \%$, without statistical difference. Higher long-term recurrence rate may be due to incomplete embolization, revascularization, or recanalization.

In the comparison of the conservative treatment, bronchial artery embolization, surgical treatment, and the concurrent treatment of bronchial artery embolization and surgery, short-term control showed the highest success rate with bronchial artery embolization, which is in agreement with previously reported results. However, surgical treatment was more efficient than conservative treatment, indicating that it might be indicated for patients with clinically stabilized condition. The reason that the concurrent treatment group of bronchial artery embolization and surgical treatment was low in its success rate was that it failed in controlling bleeding, resulting in higher morbidity and mortality due to hypovolemic shock or pulmonary dysfunction.

As for the long-term control, no differences were found between groups. Since a considerable number of hemoptysis patients had pulmonary dysfunction due to chronic lung diseases, such as tuberculosis, and had concomitant chronic diseases, such as diabetes and hypertension, they did not show any difference in the long-term observation.

Kim et al. ${ }^{11}$ described underlying lung disease and amount of bleeding as reliable risk factors for the recurrence, in a study involving 75 patients with a result estimating $54.5 \%$ of rebleeding rate after 3 years. In this study, the amount of hemoptysis had some statistical relation with the recurrent event and lung cancer tended to have more recurrence rate compared to other causes.

In the comparison of hospitalization duration by group, conservative treatment group was the shortest among other groups. This is an expected result in that the patients who are clinically stabilized and have a small amount of hemoptysis would first try conservative treatment. However, the short-term success rate was higher in the BAE group or surgical treatment group than conservative treatment group. This can be attributed to the fact that the observation period increases with the type of treatment, and not with the improvement of hemoptysis.

This study has several limitations, particularly its retrospective design. Furthermore, the evaluation and therapeutic decisions were not based on a preset protocol but rather on the clinical experience of each attending physician. It was conducted in a referral center with extensive experience with hemoptysis, which may limit the generalization of our results. A referral center may select the most severe patients, who are presumed to have a worst prognosis. Conversely, a referral center may certainly induce a better management, in terms of resource facilities, therefore contributing to a better prognosis.

This study is of significance because no previous study has comprehensively investigated the cause of hemoptysis, and not many studies have compared the efficacy and outcomes of currently available treatment methods for hemoptysis. 
BR Lee et al: Analysis of patients with hemoptysis in a tertiary referral hospital

\section{References}

1. Kim SK. Differential diagnosis of hemoptysis. Korean J Med 1998;55:634-41.

2. Yoon W, Kim JK, Kim YH, Chung TW, Kang HK. Bronchial and nonbronchial systemic artery embolization for life-threatening hemoptysis: a comprehensive review. Radiographics 2002;22:1395-409.

3. Knott-Craig CJ, Oostuizen JG, Rossouw G, Joubert JR, Barnard PM. Management and prognosis of massive hemoptysis: recent experience with 120 patients. J Thorac Cardiovasc Surg 1993;105:394-7.

4. Santiago S, Tobias J, Williams AJ. A reappraisal of the causes of hemoptysis. Arch Intern Med 1991;151:244951.

5. Fernando HC, Stein M, Benfield JR, Link DP. Role of bronchial artery embolization in the management of hemoptysis. Arch Surg 1998;133:862-6.

6. Fidan A, Ozdoğan S, Oruç O, Salepçi B, Ocal Z, Cağlayan B. Hemoptysis: a retrospective analysis of 108 cases. Respir Med 2002;96:677-80.

7. Ayed A. Pulmonary resection for massive hemoptysis of benign etiology. Eur J Cardiothorac Surg 2003;24: 689-93.

8. Crocco JA, Rooney JJ, Fankushen DS, DiBenedetto RJ, Lyons HA. Massive hemoptysis. Arch Intern Med 1968; 121:495-8

9. Erdogan A, Yegin A, Gürses G, Demircan A. Surgical management of tuberculosis-related hemoptysis. Ann Thorac Surg 2005;79:299-302.

10. McCollun WB, Mattox KL, Guinn GA, Beall AC Jr. Immediate operative treatment for massive hemoptysis. Chest 1975;67:152-5.

11. Kim SO, Oh IJ, Kim KS, Yu YK, Lim SC, Kim YC, et al. Recurrent hemoptysis after bronchial artery embolization. Tuberc Respir Dis 2001;51:364-72.
12. Swanson KL, Johnson CM, Prakash UB, McKusick MA, Andrews JC, Stanson AW. Bronchial artery embolization: experience with 54 patients. Chest 2002;121:78995.

13. Chung WY, Byun MK, Park MS, Hahn CH, Kang SM, Lee DY, et al. Risk factors of recurrent hemoptysis after bronchial artery embolization. Tuberc Respir Dis 2006; 60:65-71.

14. Rémy J, Arnaud A, Fardou H, Giraud R, Voisin C. Treatment of hemoptysis by embolization of bronchial arteries. Radiology 1977;122:33-7.

15. Yeo DS, Lee SY, Hyun DS, Lee SH, Kim SC, Choi YM, et al. Effect of bronchial artery embolization (BAE) in management of massive hemoptysis. Tuberc Respir Dis 1999;46:53-64.

16. Abal AT, Nair PC, Cherian J. Haemoptysis: aetiology, evaluation and outcome: a prospective study in a third-world country. Respir Med 2001;95:548-52.

17. Mayr B, Ingrisch H, Häussinger K, Huber RM, SunderPlassmann L. Tumors of the bronchi: role of evaluation with CT. Radiology 1989;172:647-52.

18. Hirshberg B, Biran I, Glazer M, Kramer MR. Hemoptysis: etiology, evaluation, and outcome in a tertiary referral hospital. Chest 1997;112:440-4.

19. Strickland B. Investigating haemoptysis. Br J Hosp Med 1986;35:242, 246-51.

20. Brinson GM, Noone PG, Mauro MA, Knowles MR, Yankaskas JR, Sandhu JS, et al. Bronchial artery embolization for the treatment of hemoptysis in patients with cystic fibrosis. Am J Respir Crit Care Med 1998;157(6 Pt 1):1951-8.

21. Mal H, Rullon I, Mellot F, Brugière O, Sleiman C, Menu $\mathrm{Y}$, et al. Immediate and long-term results of bronchial artery embolization for life-threatening hemoptysis. Chest 1999;115:996-1001. 